\title{
Analysis of Freeway Safety Influencing Factors on Driving Workload and Performance Based on the Gray Correlation Method
}

\author{
Lian Xie, ${ }^{1,2}$ Chaozhong Wu, ${ }^{1,3}$ Min Duan, ${ }^{4}$ and Nengchao Lyu $\mathbb{D}^{1,3}$ \\ ${ }^{1}$ Intelligent Transportation Systems Research Center, Wuhan University of Technology, Wuhan 430063, China \\ ${ }^{2}$ School of Architecture and Transportation Engineering, Guilin University of Electronic Technology, Guilin 541004, China \\ ${ }^{3}$ National Engineering Research Center for Water Transport Safety, Wuhan 430063, China \\ ${ }^{4}$ Teaching Department of Public Courses, Hunan Communication Polytechnic, Changsha 410132, China
}

Correspondence should be addressed to Nengchao Lyu; lnc@whut.edu.cn

Received 23 June 2021; Revised 23 July 2021; Accepted 25 August 2021; Published 9 September 2021

Academic Editor: Xinqiang Chen

Copyright ( 92021 Lian Xie et al. This is an open access article distributed under the Creative Commons Attribution License, which permits unrestricted use, distribution, and reproduction in any medium, provided the original work is properly cited.

\begin{abstract}
Human-related factors are a crucial inducement of traffic accidents. Understanding the influence of freeway environments on the driving behavior and workload experienced by drivers has been demonstrated to be of primary importance for improving traffic safety. To study the effect of alignment, traffic flow, and sign information on drivers' mental workload and behavior, 16 scenarios were constructed using the orthogonal design method, and simulated driving experiments were carried out with 45 participants. During driving, indicators such as the mean and standard deviation of vehicle speed and lane departure were collected, and the NASA-TLX questionnaire was adopted to measure workload. Analysis of variance results indicated that the radius of the horizontal curve, gradient, flow, and sign information level have a significant influence on drivers' workload and speed keeping ability. In addition, the horizontal curve radius has a significant effect on lane keeping ability. The importance of safety influencing factors on driving workload and performance was quantitatively ranked by integrating the trend of Deng's correlation degree, comprehensive correlation degree, and similar correlation degree, whose weight was calculated using the entropy method. Traffic sign information was found to have the greatest impact on workload. In terms of driving performance, traffic volume has the greatest influence on the mean and standard deviation of vehicle speed, followed by the amount of sign information. Lane departure is most affected by the radius of the horizontal curve. These findings provide guidance for freeway traffic safety regulation, including workload control and road facility optimization.
\end{abstract}

\section{Introduction}

Statistics of road traffic accidents show that $80 \%$ of accidents and $65 \%$ of dangerous driving conditions are directly or indirectly caused by human factors, and that the driver has no obvious illegal and subjective errors in $41 \%$ of these accidents [1]. In 1988, John [2] proposed the concept of mental workload, also known as mental load or cognitive load, and noted that the cognitive process of information will consume the memory resources of the brain and cause a certain workload. Extended to the field of transportation, driving workload is considered to be a measure of mental resources occupied by a series of activities related to driving tasks, such as receiving external stimuli, rapidly processing information and forming a clear judgment [3]. Mental workload can be defined as the ratio between the capacity of the information processing system needed to correctly perform the task and the amount of available attentional resources [4]. From the driver's point of view, every driving event provokes a specific level of workload depending on its complexity and the road environment, such as road design, road layout, and traffic flow [5].

Mental workload is a multifaceted concept, and the measures available for the assessment of workload can be grouped into three categories: subjective, physiological, and performance. Subjective measures rely on the assumption 
that workload effectively experienced by consulted drivers is the most accurate judgment, which is collected after the task without interference to the driving. One of the most common self-report questionnaires is the NASA-Task Load Index (NASA-TLX) [6]. Physiological indexes can be realized continuously through online evaluation of driving workload based on the real-time observation through recording medical device that comprises blood pressure, pulse, heart rate, skin temperature, galvanic skin, EEG, and other indicators [7]. Performance measures refer to drivers' operation behavior as an alternative indicator of mental load, which potentially reflects the state of stress. Driving behavior measurements include not only the analysis of the primary task indicator but also the investigation of secondary activities [8]. In general, the primary task measurement mainly focuses on the driver's operating characteristics for completing driving tasks in specific scenarios. In previous simulation driving research, indicators such as vehicle speed, reaction time, and the number of driver mistakes are often used to measure performance. In field tests, characteristics such as vehicle trajectory, steering wheel, accelerator, and brake pedal control are considered [9]. Secondary task measurement is often used when the driver needs to complete multiple tasks. There are two main paradigms: task loaded and subordinate task. Neng-Chao et al. [10] proposed quantifying direction sign information via information entropy theory and used different signs to load driving workload, which was verified to be effective. Baldauf et al. [11] studied the feasibility of perception time as a driving workload evaluation index. Experiments show that the more complex the driving environment, the longer the driver's reaction time to peripheral tasks, and the higher the workload experienced.

Driving performance can not only be used as an evaluation index of workload [12] but also interact with driving workload, because the perception and information transmission of the road environment runs throughout the driving process. To quantify the relationship between driving workload and behavior, Ryu and Myung [13] proposed a single-resource model. When the task demand increases, the central nervous system increases the resource supply so that the task can be better completed. When all psychological events share the same cognitive resources, each individual has a limited ability to process information. Based on the single-resource model, Wiberg et al. [14] believe that there are different types of resources, such as visual channels, auditory channels, information processing, and response execution. If several tasks need to use the same channel resources, the workload will increase. Consistent with the Yerkes-Dodson law, Zokaei et al. [15] tracked drivers' physiological response and behavior under different workload levels and found that to maintain the best driving performance the workload should not be too high or too low.

Although driving workload and driving performance are affected by the individual differences, strategies, and emotions of drivers [16], the impact of the external traffic environment, which includes road alignment, flow, traffic facilities, etc., is of greater concern to scholars. Among these factors, road geometry undoubtedly plays a key role.
Complex road alignments, such as curves or slopes, have been shown to demand more driver attention [17]. As the uncertainty of the road ahead increases due to insufficient viewing distance, drivers must allocate more attention resources to collecting information and decision making, which results in an increased driving workload [18]. Tight horizontal radius curves associated with abrupt grade changes, as well as compound curves, were identified as a threat to the controllability of cars and can contribute to severe crashes [19]. The driver must adjust the vehicle speed and lane offset to adapt to the alignment changes and even use corner cutting action or continuous pedal control to regulate the engine's output power and braking force to maintain driving stability [20]. Considering that pedal operation on steep downhill sections can easily lead to an increase in mental workload, $\mathrm{Hu}$ et al. [21] proposed designing the definition standard of long and steep sections based on driving demand to design long and vertical slope sections from the driving needs to define standards, which is conducive to improve the driving performance. In contrast, few geometric changes combined with repetitive and stimulus-free environments create a monotonous driving experience that can lead to mind wandering and task disengagement and subsequently affect drivers' performance [22].

It is widely known that there are limitations to the amount of information humans can process; the more information displayed to a driver at one time, the more difficult it is for the driver to accurately process all of the information [23]. Studies have shown that drivers' recognition time increases with increasing sign information, and when the sign information reaches a certain threshold, the recognition time will increase significantly [24]. Multiboard signs present information more dispersedly, which requires the drivers to search for longer periods of time to find the target, which undoubtedly increases the task processing demand [25]. In addition to a longer reaction time, a complex visual environment will lead to a higher workload and lower driving speed, as shown in simulated driving conditions [26]. According to the comprehensive efficiency of the cognitive response of road signs, the number of place name thresholds of highway direction signs should be 6 to 7 $[25,27]$.

Traffic density is an important indicator of traffic complexity. With an increase in traffic flow, drivers' workload shows a linear upward trend until the level of medium traffic flow. When lane change occurs in front of the driver's field of vision, the driving workload increases; the closer the distance, the greater the workload [28]. Similarly, Fitzpatrick [29] found that compared with free driving, traffic congestion will lead to an increased driving workload based on an analysis of drivers' attention and reaction time to the deceleration of the car in front. In some special road sections, such as ramps, vehicle interleaving conflict increases drivers' lane offset and deteriorates lane keeping capacity, resulting in increased driving load [30]. Meanwhile, an increase in the number of heavy trucks will lead to an increase in the psychological load of adjacent drivers and an increase in safe distances between vehicles [31]. 
Previous studies have shown that during driving, drivers' situational awareness, decision making, and vehicle operating capacity are limited [14]. Higher driving demands were found to increase subjective workload levels and impair performance, particularly through behaviors associated with attention [32]. The task capability interface model [33] poses that the loss of control may occur when the demand of the driving task is greater than the ability of the driver because driver attention is probably detached from context driving tasks, such as situational awareness, which results in perception, decision making, and operational errors. Conversely, drivers who are in a state of low workload for long periods of time are prone to drowsiness or distraction. Both high and low levels of mental workload have been shown to lead to inaccurate perceptions, low levels of attention, and insufficient time for accurate information processing, which are the main causes of road collisions [34]. Therefore, it is of great significance to clarify the influence of driving environment factors on mental workload and performance to reduce traffic accidents and improve driving safety.

Road alignment, sign information, and traffic flow are the most common components of freeways, and they can interact and create complex traffic situations. Although the impact of the external road environment on drivers has been widely considered by scholars, there are few studies comparing the influencing factors. Currently, it is difficult to determine how and which factors profoundly affect driving workload and behavior. Therefore, the purpose of this study was to study the influence of various road environmental factors on drivers' psychology and behavior on the basis of previous research [35]. In current study, the $L_{16}\left(4^{5}\right)$ orthogonal experimental design was used to select representative points from the overall experiment, which reduced the number of experiments and resulted in a small data sample, and the reflected information was inaccurate and incomplete, with gray characteristics. Meanwhile, in view of the disadvantage that the $L_{16}\left(4^{5}\right)$ orthogonal test cannot analyze the interaction of various factors, the gray correlation method has been proposed to quantitatively evaluate the influence of the horizontal curve radius, longitudinal slope, traffic flow, and sign information on driving workload and performance, which is suitable for the analysis of uncertainty problems with the characteristics of small sample size, irregular data, lack of information, and insufficient experience and has been widely used in the analysis of influencing factors in various fields [36].

\section{Materials and Methods}

The supplementary experiment was carried out on the basis of a previous study [35]. The number of subjects increased from 11 to 45 in this research. More details of the experimental design can be found in a previous study.

\subsection{Experiment Design and Procedure}

2.1.1. Apparatus. The driving simulation platform developed by the Intelligent Transportation Systems Research Center of Wuhan University of Technology was used to conduct the experiment and collect the data, which were composed of an instrumented vehicle and UC-win/road software, as shown in Figure 1.

2.1.2. Subjects. Through the supplementary experiment, the number of subjects was increased to 45 due to equipment failure, wherein one person data sample was removed, and finally, a total of 44 subjects' data were collected. Among them, 39 are males and 5 are females. Participants ranged in age from 22 to 62 years $(M=31.07 \pm 9.78)$; participants had held a valid driver's license between 2 and 28 years $(M=6.80 \pm 6.37)$ and drove between 2 and 100 thousand kilometers per year $(M=15.7 \pm 19.0)$.

2.1.3. Scenario Design. The present study focused on the effect of radius, slope grade, traffic flow, and sign information on driving workload and performance. Taking into account the different levels of each factor, an orthogonal test was designed according to the $L_{16}\left(4^{5}\right)$ orthogonal table, which was used to reduce the number of trials. Finally, 16 simulated driving test scenarios were designed. More details of the scene design parameters can be found in [35].

2.1.4. Procedure. Prior to testing, participants were asked to read and sign an informed consent form, fill out a personal information questionnaire, and confirm that they were in good health and had no serious disease or serious accident experience. By explaining the meaning of the NASA-TLX scale and each question, we confirmed that each subject could accurately evaluate the degree of driving workload experienced under different scenarios after adaptation exercises, which made them proficient in driving tasks. At the same time, the participants were confirmed to have no vertigo or other uncomfortable reactions through a pre-experiment.

Then, irrelevant personnel were required to leave the simulation cab and turn off all communication tools to keep the room quiet. The subjects were reminded to choose a comfortable driving posture and to maintain their driving habits. Data acquisition began at this time. Before entering the test section, experiment coordinators told the subjects the destination and asked them to state the driving direction of the destination when driving past the guide signs to ensure that subjects were able to complete visual recognition of the sign. After completing a test scene, the experiment coordinator assisted the subjects in finishing the NASA-TLX scale for the scene through oral questions.

To avoid the learning effect, driving scenarios were randomly sorted. When all 16 test scenarios were completed, the subjects were required to fill in the item paired comparison table of the NASA-TLX scale. Finally, the data collection was turned off, and the data were stored.

2.1.5. Data Collection. In this study, two categories of data were collected: subjective workload and vehicle motion. The NASA-TLX questionnaire was used to measure drivers' subjective workload, which has been proven to be reliable for measuring different workload levels [37]. After completing a 

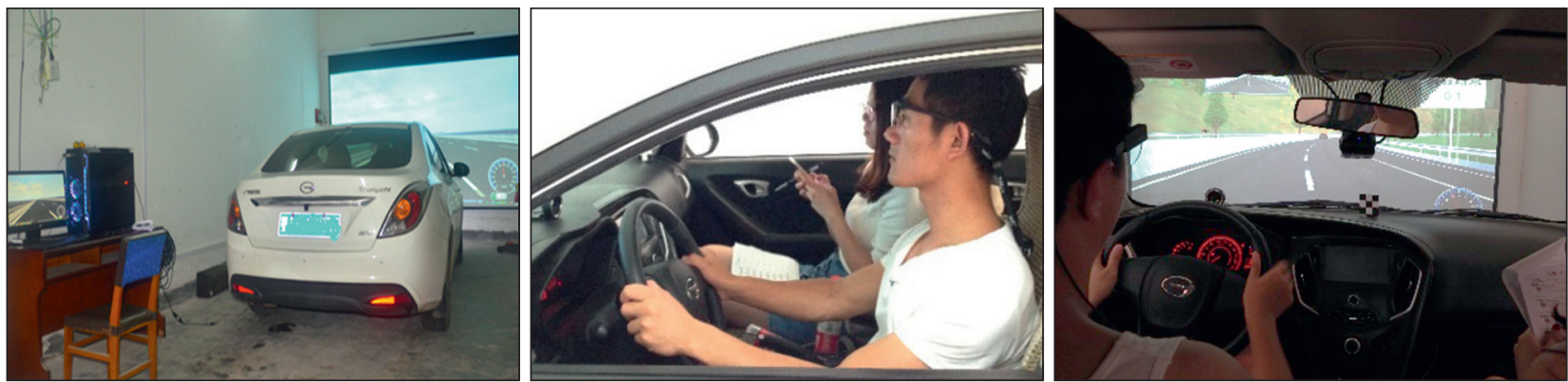

FIGURE 1: Driving simulator and scene.

certain test section, participants were asked to rate their workload using the NASA-TLX questionnaire, which consists of six subscales of workload (10-point rating scale): mental demand, physical demand, temporal demand, performance, effort, and frustration. The overall NASA-TLX workload score was calculated by multiplying each dimension by the corresponding rating.

During the experiment, operating data (steering, gas, brake, etc.), vehicle motion data (speed, acceleration, yaw rate, etc.), and maneuver data (time headway, lane deviation, etc.) were collected in real time at a frequency of $10 \mathrm{~Hz}$. As performance measures, driving speed and lane deviation were derived for each participant, which indicated the driver's longitudinal and lateral control performance, respectively [38].

2.2. Gray Correlation Analysis Method. The basis of gray correlation analysis is to determine whether a comparison sequence is closely related to a reference sequence according to the degree of similarity of the geometric shape of the sequence curve.

2.2.1. Data Sequence and Dimensionless. The purpose of this study is to evaluate the effects of the horizontal curve radius, longitudinal slope, traffic flow, and sign information on driving workload and performance. Therefore, in this paper, the subjective workload, the mean, and standard deviation of vehicle speed and lane offset are used as reference sequences $\left\{x_{0}(t)\right\}(t=1,2, \ldots)$. The horizontal curve radius, longitudinal slope, traffic flow, and sign information are taken as comparison sequences, namely, $\left\{x_{1}(t)\right\},\left\{x_{2}(t)\right\},\left\{x_{3}(t)\right\}$, and $\left\{x_{4}(t)\right\}$.

Due to the different units of the data sequence of each factor, it is necessary to standardize the original data to eliminate the influence of dimensions. The conversion formula is as follows:

$$
X_{i}^{\prime}(k)=\frac{X_{i}(k)-\overline{X_{i}}}{\sigma},
$$

where $\overline{X_{i}}$ and $\sigma$ are the mean value and standard deviation of the data column $X_{i}^{(0)}(k)$.

2.2.2. Deng's Correlation Degree. The correlation coefficient $\left(\gamma_{0 i}(k)\right)$ represents the difference in geometric shape between the sequence curves. The difference between the curves is used as the basis to measure the correlation coefficient as

$$
\begin{array}{r}
\gamma_{0 i}(k)=\frac{\min _{i} \min _{k}\left|x_{0}^{\prime}(k)-x_{i}^{\prime}(k)\right|+\xi \max _{i} \max _{k}\left|x_{0}^{\prime}(k)-x_{i}^{\prime}(k)\right|}{\left|x_{0}^{\prime}(k)-x_{i}^{\prime}(k)\right|+\xi \max _{i} \max _{k}\left|x_{0}^{\prime}(k)-x_{i}^{\prime}(k)\right|}, \\
k=1,2, \ldots, n ; i=1,2, \ldots, m,
\end{array}
$$

where $\xi$ is the resolution coefficient, which is in the range of $(0,1)$, usually $0.5 ; \quad \min _{i} \min _{k}\left|x_{0}^{\prime}(k)-x_{i}^{\prime}(k)\right|$ and $\min _{i} \min _{k}\left|x_{0}^{\prime}(k)-x_{i}^{\prime}(k)\right|$ are the maximum and the minimum difference of the two levels, respectively.

The correlation degree $\left(\gamma_{0 i}\right)$ is expressed quantitatively by the average value of the correlation coefficient at each time of the sequence as

$$
\gamma_{0 i}=\frac{1}{n} \sum_{k=1}^{n} \gamma_{0 i}(k), \quad i=1,2, \ldots, m,
$$

where $\gamma_{0 i}$ is the correlation degree between the comparison sequence and the reference sequence and $n$ is the length of the comparison sequence.

2.2.3. Synthesis Correlation Degree. Suppose the sequences $X_{i}$ and $X_{j}$ have the same length and the initial value is not equal to zero; $\varepsilon_{i j}$ and $r_{i j}$ are the absolute correlation degree and relative correlation degree of $X_{i}$ and $X_{j}$, respectively, and $\theta \in[0,1]$; then, $\rho_{i j}$ is the gray synthesis correlation degree of $X_{i}$ and $X_{j}$, which is

$$
\rho_{i j}=\theta \varepsilon_{i j}+(1-\theta) r_{i j} .
$$

The synthesis correlation degree not only reflects the similarity degree of broken lines $X_{i}$ and $X_{j}$ but also reflects the closeness of the change rate of $X_{i}$ and $X_{j}$ relative to the starting point, which is a quantitative index to comprehensively characterize the relationship between sequences. Generally, it is suggested to use $\theta=0.5$.

2.2.4. Similarity Correlation Degree. Suppose the sequences $X_{i}$ and $X_{j}$ have the same length, $\varepsilon_{i j}$ is the gray correlation degree of $X_{i}$ and $X_{j}$ based on the similarity perspective, which is calculated as 


$$
\varepsilon_{i j}=\frac{1}{1+\left|s_{i}-s_{j}\right|},
$$

where $s_{i}-s_{j}=\int_{1}^{n}\left(X_{i}^{0}-X_{j}^{0}\right) \mathrm{d} x$. In the integral, the integrand $X_{i}^{0}-X_{j}^{0}$ is a broken line formed by the sequence $X_{i}^{0}-$ $X_{j}^{0}$ connecting the adjacent nodes.

The similarity correlation degree is used to measure the geometric similarity of sequences $X_{i}$ and $X_{j}$. The more similar the geometry, the greater the value of $\varepsilon_{i j}$, and vice versa.

\subsubsection{Comprehensive Evaluation Based on Entropy Weight} Method. Due to the problem of the fluctuation of correlation coefficient in the calculation method of gray correlation degree having an effect on the calculation results of correlation degree [39], the weighted average of Deng's correlation degree, comprehensive correlation degree and similar correlation degree is carried out based on entropy weight method, and the comprehensive evaluation model of correlation degree are established.

Entropy has its origins as a concept in thermodynamics and is a measure of the disorder degree of the system. Information entropy is often used to measure the degree of order of the system state. The entropy weight method uses the size of the effective amount of information contained in the data to measure the impact of each indicator on the comprehensive evaluation [40]. The smaller the information entropy of the evaluation index, the greater the amount of information provided, and the greater the role it plays in the comprehensive evaluation, the greater the weight of the index.

According to the definition of information entropy, the calculation is as follows:

$$
E_{j}=-\frac{1}{\ln n} \sum_{i=1}^{n}\left(p_{i j} \ln _{p_{i j}}\right),
$$

where $p_{i j}=y_{i j} / \sum_{j=1}^{m} y_{i j}$ and $y_{i j}$ are the different types of correlation degree values for influencing factors. If $p_{i j}=0$, then $p_{i j} \ln _{p_{i j}}=0$.

The weight of each correlation index is calculated by information entropy as follows:

$$
w_{j}=-\frac{\left(1-E_{j}\right)}{m-\sum_{j=1}^{m} E_{j}} .
$$

\section{Results}

3.1. Subjective Workload Measurements. The distribution of subjective workload scores under different test scenarios is shown in Figure 2. There was a significant difference for each scenario $(F \quad(15,688)=18.719, p<0.001)$. The average workload score was between 28.9 and 62.5. Among the scenarios, the driver's workload on the $9^{\text {th }}$ section was the highest, reaching 62.5, which consisted of a sharp curve $(R=300 \mathrm{~m})$, steep slope $(i=-5.5 \%)$, large traffic flow $(1400 \mathrm{pcu} / \mathrm{h} \bullet \mathrm{ln})$, and greater sign information (199 bit). Not only is the perception and processing load of environmental information large, but vehicle handling is also complex in sharp curves and steep slope sections. In contrast, the minimum average workload value of 28.9 appeared on the $16^{\text {th }}$ test section, which contained gentle road alignment and low traffic flow and sign information.

3.2. Driving Performance Measurements. The distribution of the mean and standard deviation of vehicle speed under different test scenarios is shown in Figure 3. The mean value of vehicle speed $(F(15,688)=11.052, p<0.001)$ and standard deviation of vehicle speed (Brown Forsythe and Welch corrected $p<0.001$ ) are significantly different in each scenario. From the average speed, the speed of the sharp curve section with a small horizontal curve radius (such as scene $1,7,9$, and 11) is significantly reduced. For speed stability, the largest average value of the standard deviation of each driver occurs in the $9^{\text {th }}$ test section, and the change range of the standard deviation of the speed is also the largest, indicating that different drivers show different speed stabilities in this complex section with sharp curves and steep slopes.

The mean value and standard deviation of lane deviation under different test scenarios are shown in Figure 4. There were significant differences in the mean and standard deviation of lane departure under each scenario (Brown Forsythe and Welch corrected $p<0.001$ ). Although the vehicle speeds in sharp curve sections (such as scenes 1, 7, 9, and 11) are significantly reduced, the lane offset is relatively high. In addition to the mean value of lane offset increasing, the standard deviation of lane offset is also relatively high. However, the fluctuation range of the standard deviation of lane offset is large, especially in the $9^{\text {th }}$ and $7^{\text {th }}$ test sections, which indicates that subjects show different lane keeping abilities in specific test sections.

\subsection{Effect of Factors on Workload and Performance}

3.3.1. Results of Multivariate Analysis of Variance. The results of multivariate analysis of variance of the orthogonal test are listed in Table 1. The table shows that the radius of the horizontal curve had a significant effect on drivers' workload, vehicle speed, and lane keeping ability $(p<0.001)$. Slope and sign information had a significant effect on drivers' workload and speed keeping ability $(p<0.05)$. Although there was not a significant difference in the mean value of lane offset $(p=0.935, p=0.342)$, the standard deviation of lane offset showed a significant difference $(p<0.05)$. Traffic flow only had a significant effect on subjective workload and vehicle speed $(p<0.05)$, and the mean and standard deviation of lane offset were not significantly different $(p=0.607, p=0.506)$. Although variance analysis can analyze the influence of traffic environment factors on workload and driving behavior, it cannot quantitatively evaluate the degree of influence for each factor and determine the importance of different influencing factors. 


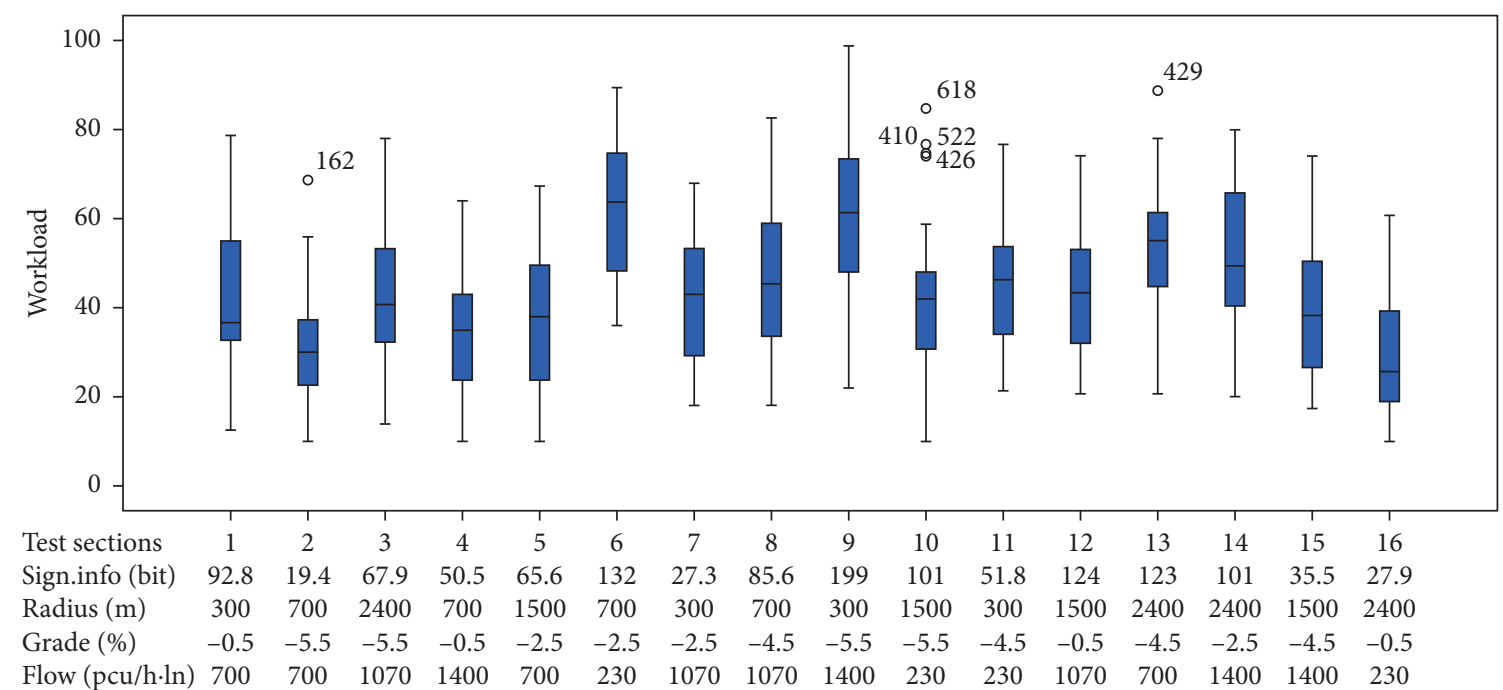

Figure 2: Subjective workload for each test section.

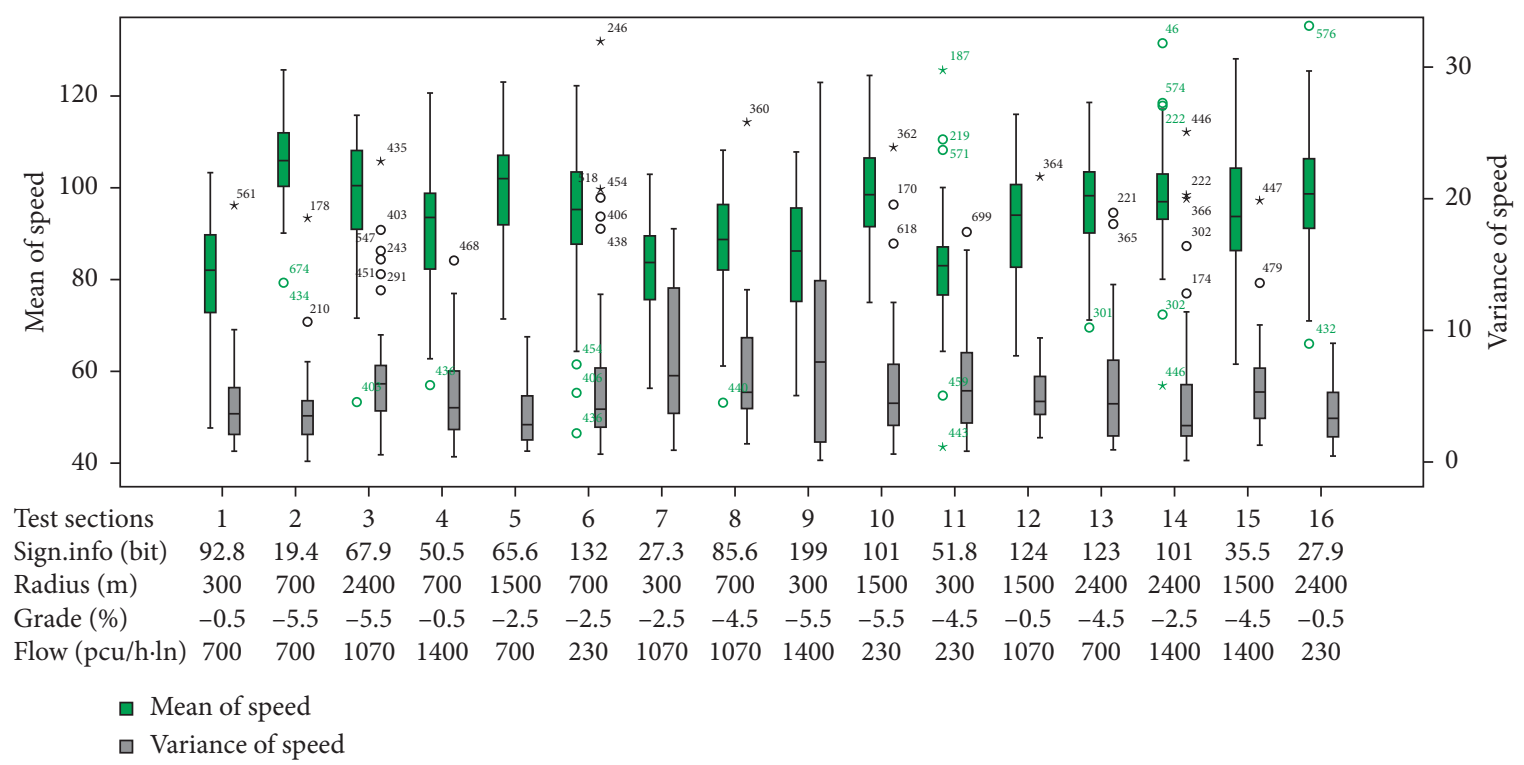

Figure 3: Mean and variance of vehicle speed for each test section.

3.3.2. Results of Gray Correlation Analysis. Three gray correlation models, namely, Deng's correlation degree, synthesis correlation degree, and similarity correlation degree, were used to calculate the correlation coefficients between various factors of the driving environment and the workload and performance. Based on the entropy weight method, the weight of each correlation method is determined, and the workload and driving performance influencing factors were comprehensively evaluated and ranked, as shown in Table 2.

The comprehensive evaluation values of the correlation degree corresponding to the sign information, flow, slope, and radius are $0.892,0.712,0.575$, and 0.566 , respectively. It is concluded that the amount of sign information has the greatest impact on the driver's workload. The volume of traffic flow has the greatest influence on the mean and standard deviation of vehicle speed, with comprehensive evaluation values of 0.809 and 0.773 , respectively, which were higher than the correlation degree of other factors. The radius of the horizontal curve has the greatest impact on the mean and standard deviation of lane offset, and the comprehensive evaluation values were 0.849 and 0.874 , respectively.

\section{Discussion}

The objective of this study was to supplement the previous study and further quantify and rank the influencing factors of driving workload and performance, including radius, slope, flow, and sign information. To this aim, the simulation experiment was carried out through an orthogonal experimental design, and the driver's subjective workload, speed, 


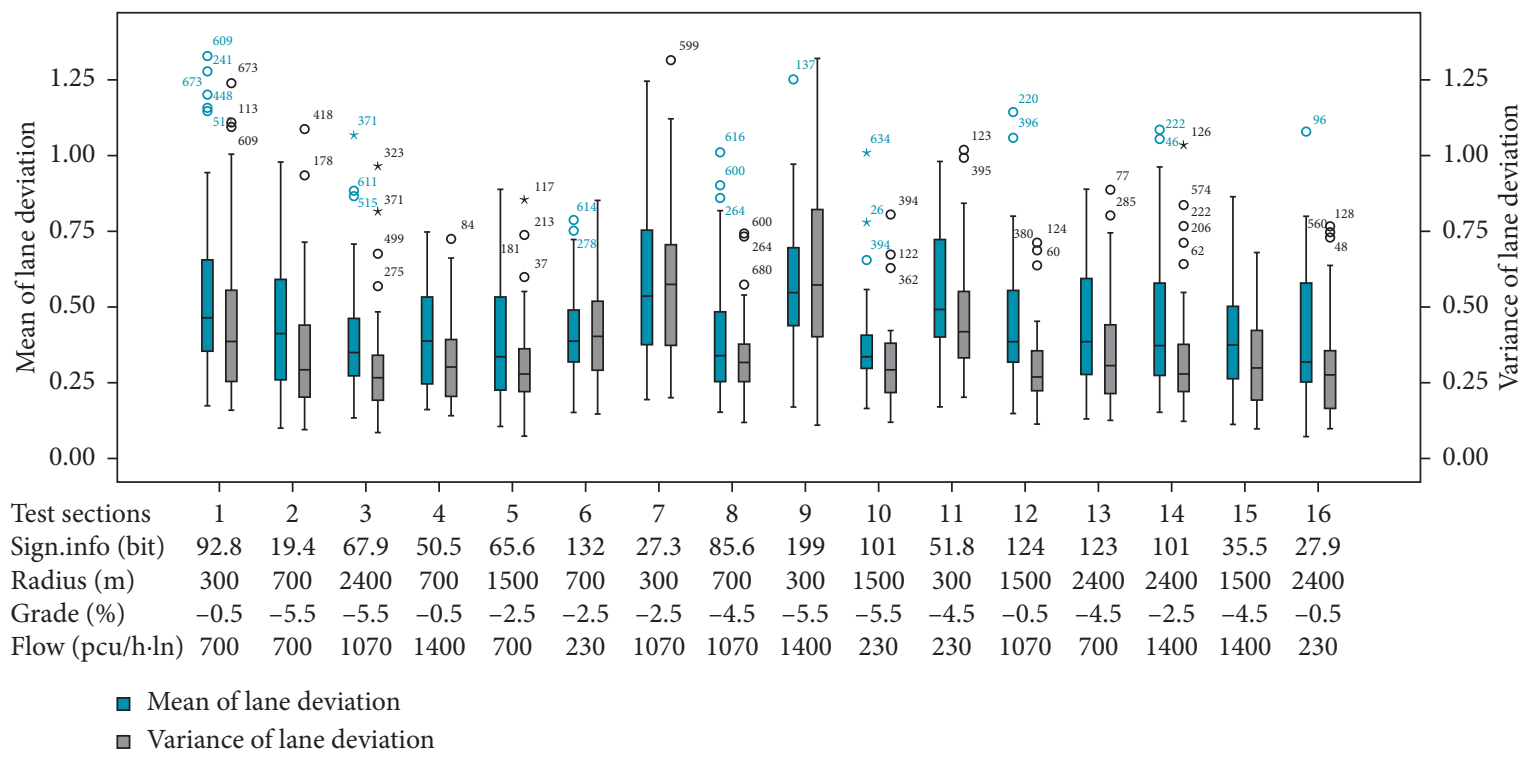

Figure 4: Mean and variance of lane deviation for each test section.

TABLE 1: MANOVA results for subjective workload and driving performance.

\begin{tabular}{|c|c|c|c|c|c|c|}
\hline Source & Indicators & Type III sum of squares & $\mathrm{df}$ & Mean square & $F$ & Sig. \\
\hline \multirow{5}{*}{ Radius } & Workload & 5859.036 & 3 & 1953.012 & 8.798 & 0.000 \\
\hline & Mean of speed & 21576.295 & 3 & 7192.098 & 37.471 & 0.000 \\
\hline & Variance of speed & 372.335 & 3 & 124.112 & 5.876 & 0.001 \\
\hline & Mean of lane deviation & 3.001 & 3 & 1.000 & 22.646 & 0.000 \\
\hline & Variance of lane deviation & 5.515 & 3 & 1.838 & 53.026 & 0.000 \\
\hline \multirow{5}{*}{ Grade } & Workload & 10812.170 & 3 & 3604.057 & 16.235 & 0.000 \\
\hline & Mean of speed & 3626.594 & 3 & 1208.865 & 6.298 & 0.000 \\
\hline & Variance of speed & 342.024 & 3 & 114.008 & 5.398 & 0.001 \\
\hline & Mean of lane deviation & .019 & 3 & 0.006 & 0.141 & 0.935 \\
\hline & Variance of lane deviation & .457 & 3 & 0.152 & 4.394 & 0.004 \\
\hline \multirow{5}{*}{ Flow } & Workload & 2917.339 & 3 & 972.446 & 4.380 & 0.005 \\
\hline & Mean of speed & 3093.921 & 3 & 1031.307 & 5.373 & 0.001 \\
\hline & Variance of speed & 595.479 & 3 & 198.493 & 9.398 & 0.000 \\
\hline & Mean of lane deviation & .081 & 3 & 0.027 & 0.612 & 0.607 \\
\hline & Variance of lane deviation & .081 & 3 & 0.027 & 0.779 & 0.506 \\
\hline \multirow{5}{*}{ Sign information } & Workload & 40714.972 & 3 & 13571.657 & 61.135 & 0.000 \\
\hline & Mean of speed & 2248.253 & 3 & 749.418 & 3.905 & 0.009 \\
\hline & Variance of speed & 175.308 & 3 & 58.436 & 2.767 & 0.041 \\
\hline & Mean of lane deviation & .148 & 3 & 0.049 & 1.116 & 0.342 \\
\hline & Variance of lane deviation & .473 & 3 & 0.158 & 4.548 & 0.004 \\
\hline
\end{tabular}

lane offset, and other performance indicators were collected. Based on the entropy weight method, three different correlation degree models were comprehensively evaluated.

4.1. Influence of Factors on Subjective Workload. As expected, a higher mental workload subjective estimation (NASA-TLX questionnaire) was obtained for the complex experimental scenario compared to the simple experimental scenario. Thus, different levels of driving environment and task demand lead to corresponding self-estimated workload in line with previous studies [4]. Further, Neng-Chao et al. [10] confirmed that the driving workload can be loaded through the designed driving environment and is controllable by researching the relationship between environmental factors and driving workload indexes.

A ranking of four factors that affect the driver's workload is shown in Figure 5, based on the comprehensive evaluation of the entropy weight method. As shown, three types of gray correlation results have similar trends. The amount of sign information has the greatest impact on drivers' workload, followed by traffic volume, according to the result that $r_{S}>r_{F}>r_{R}>r_{I}$. Analyzing the six items of the NASA-TLX questionnaire, as the amount of information on the signs increases, the mental demand and temporal demand were found to increase during driving, and the degree of effort 
TABLE 2: Correlation degree between dependent variables and influencing factors.

\begin{tabular}{|c|c|c|c|c|c|}
\hline Indicators & Model & Radius (R) & Grade (I) & Flow $(\mathrm{F})$ & Sign.info (S) \\
\hline \multirow{4}{*}{ Workload } & Deng's correlation & 0.560 & 0.677 & 0.891 & 0.939 \\
\hline & Synthesis correlation & 0.560 & 0.525 & 0.743 & 0.833 \\
\hline & Similarity correlation & 0.598 & 0.508 & 0.550 & 0.895 \\
\hline & Overall evaluation & 0.575 & 0.566 & 0.712 & 0.892 \\
\hline \multirow{4}{*}{ Mean of speed } & Deng's correlation & 0.712 & 0.575 & 0.900 & 0.904 \\
\hline & Synthesis correlation & 0.534 & 0.547 & 0.723 & 0.712 \\
\hline & Similarity correlation & 0.512 & 0.563 & 0.753 & 0.576 \\
\hline & Overall evaluation & 0.606 & 0.564 & 0.809 & 0.756 \\
\hline \multirow{4}{*}{ Variance of speed } & Deng's correlation & 0.672 & 0.565 & 0.901 & 0.880 \\
\hline & Synthesis correlation & 0.514 & 0.600 & 0.646 & 0.602 \\
\hline & Similarity correlation & 0.501 & 0.685 & 0.557 & 0.504 \\
\hline & Overall evaluation & 0.605 & 0.603 & 0.773 & 0.741 \\
\hline \multirow{4}{*}{ Mean of lane deviation } & Deng's correlation & 0.905 & 0.672 & 0.558 & 0.882 \\
\hline & Synthesis correlation & 0.620 & 0.525 & 0.542 & 0.630 \\
\hline & Similarity correlation & 0.514 & 0.500 & 0.557 & 0.501 \\
\hline & Overall evaluation & 0.849 & 0.645 & 0.556 & 0.831 \\
\hline \multirow{4}{*}{ Variance of lane deviation } & Deng's correlation & 0.890 & 0.674 & 0.572 & 0.876 \\
\hline & Synthesis correlation & 0.578 & 0.529 & 0.523 & 0.556 \\
\hline & Similarity correlation & 0.503 & 0.500 & 0.514 & 0.500 \\
\hline & Overall evaluation & 0.874 & 0.667 & 0.569 & 0.859 \\
\hline
\end{tabular}

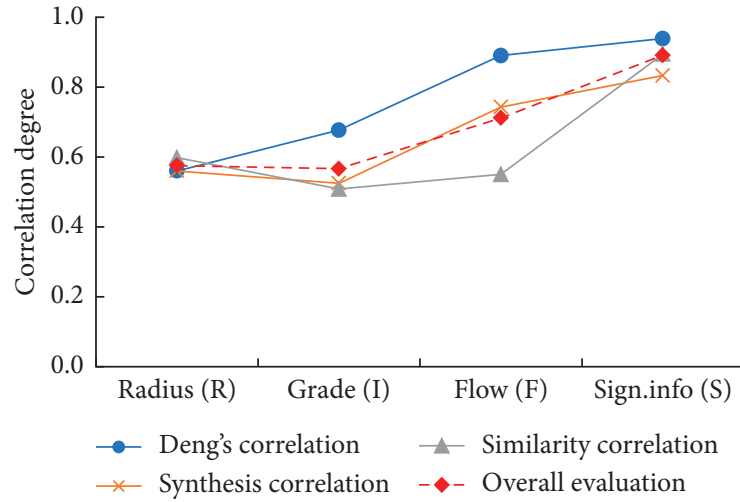

FIGURE 5: Ranking of influencing factors for workload.

invested also increases. The results indicate that the drivers' cognition of freeway directional signs will lead to increased driving workload, which is consistent with other studies [25]. On the other hand, an increase in driver effort is conducive to maintaining appropriate driving performance and achieving better sign recognition [41].

4.2. Influence of Factors on Driving Performance. As shown in Figure 6, there is a partial crossover in the three gray correlation curves between the mean and standard deviation of vehicle speed and influencing factors. The change trends of the three rankings are integrated using the entropy method, and the traffic flow and sign information are found to have a greater impact on maintaining the vehicle speed with a correlation coefficient between 0.741 and 0.809 . The slope and radius of the road alignment have a relatively small impact with a correlation coefficient between 0.564 and 0.606 . Vehicle speed and headway time are commonly used parameters for safety judgments and have attracted continuous attention from drivers. Generally, in complex scenarios, to maintain a safe headway and obtain enough time for sign recognition, drivers will adopt deceleration strategies and other compensation behaviors [42, 43]. Moreover, this indicates that driving workload has a negative impact on the longitudinal driving performance [19], the most obvious effect is a decrease in the stability of vehicle speed under high workload. A similar phenomenon was obtained in this research.

According to a ranking of the correlation degree of each influencing factor, the mean and standard deviation of lane offset are found to most affected by the radius of curve, followed by the amount of sign information (see Figure 7). Dijksterhuis et al. [44] determined that the standard deviation of the lateral position (SDLP) may show a downward trend after additional efforts were mobilized to serve the steering task when the demand for lateral control increased during driving. However, a curved road design increases the difficulty of vehicle control, which requires drivers to adopt more precise pedal and steering control to adapt to changes in curvature. When the demand exceeds the range that can be compensated by the degree of effort, the lane keeping ability decreases. Therefore, the curve is still the focus of lane lateral control optimization and sideslip and rollover accident prevention.

In conclusion, this work proposes a comprehensive evaluation based on the entropy weight method, which realizes the quantification and ranking of workload and driving performance influencing factors. The findings clarify that factors should be the focus of workload and driving behavior analysis. This can not only provide a reference for the selection of index weights for constructing a comprehensive evaluation model of traffic safety considering people-vehicles-roads-environment but also provide guidance for freeway alignment design, sign optimization, and driving workload regulation. 

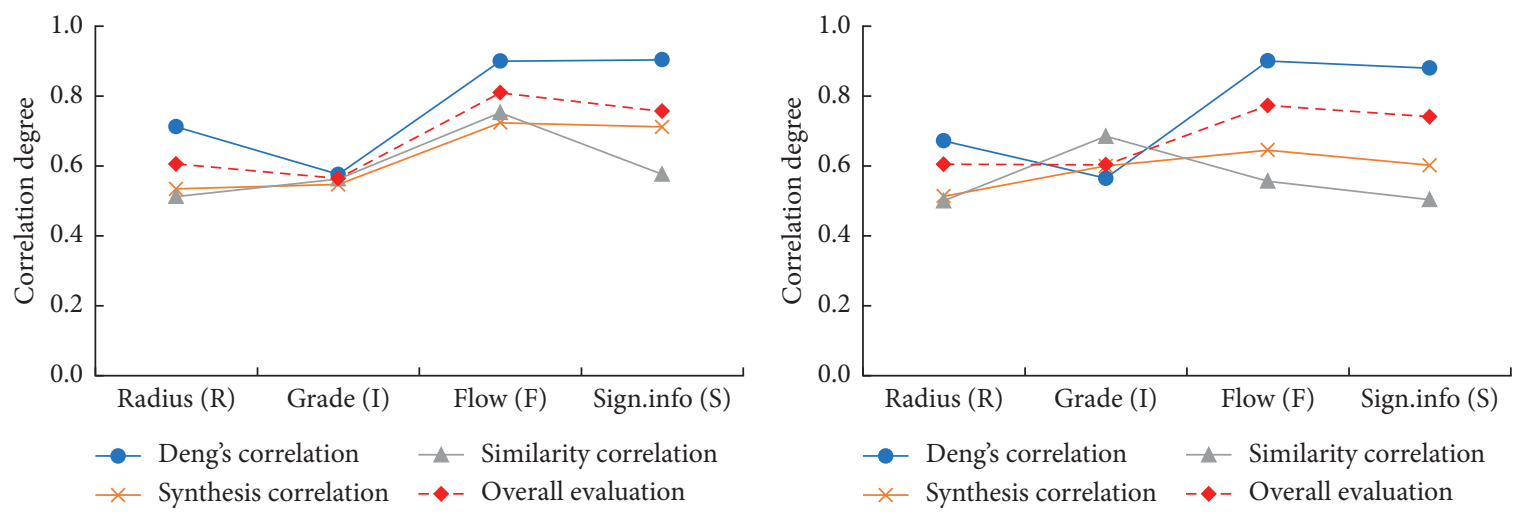

FIGURE 6: (a) Ranking of influencing factors for the mean value of vehicle speed and (b) standard deviation of vehicle speed.
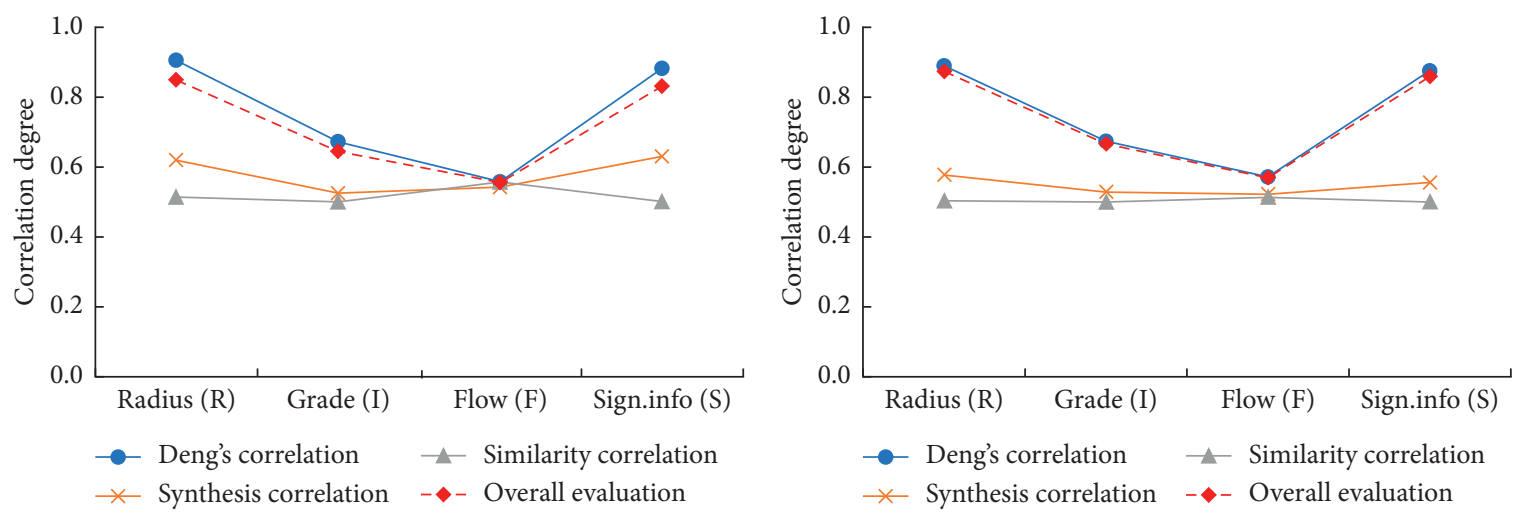

FIGURE 7: (a) Ranking of influencing factors for the mean value of lane offset and (b) standard deviation of lane offset.

4.3. Relationship between Driving Workload and Performance. It has been demonstrated that an increase in road context complexity can lead to impaired performance due to an increase in mental workload and could cause unsuitable maneuvers and even road accidents [38]. In line with the results of Figures 3 and 4, which indicated that in complex scenes, such as the $9^{\text {th }}$ section, not only does the driver experience the highest workload but also the average and amplitude of the variance of speed and lane deviation reach the maximum. However, the increased complexity of the environment may not always lead to increased driving workload or driving behavior damage. When drivers are engaged in secondary tasks, to manage workload and control driving risk, they will take strategies of interrupting, delaying, and resuming secondary tasks [45]. This may trigger drivers to adopt a compensation strategy to reduce the driving workload. For example, to obtain more time to process information for a foggy vehicle, both speed and acceleration are decreased, and the following distance of the vehicle is increased, an intentional strategy taken by the driver [23]. Fitch et al. [41] found that from a single task to multiple tasks, although the subjective workload score increased, the standard deviation of the lateral position (SDLP) decreased, which suggests that an increase in workload may improve driving performance. Similarly, it indicated that longitudinal and lateral performance parameters remain or even improve according to the task [46]. In this paper, it is found that the speed of the sharp curve section with a small horizontal curve radius (such as scenes 1, 7, 9, and 11) was significantly reduced, as shown in Figure 3, which potentially indicates that the deceleration compensation strategy has been adopted.

However, the relationship between workload and driving behavior is very complex. A large number of simulated and field driving experiments show that complex driving contexts trigger drivers to adopt compensation strategies [47]. However, it remains unclear when to take compensation behavior, the characteristics of compensation behavior, and the interaction mechanism between workload and driving behavior, which will be the focus of the author's follow-up research.

\section{Conclusion}

Through orthogonal experimental design, a simulated driving experiment with 16 test scenarios was carried out, and the influence of the radius, slope, traffic flow, and sign information factors on driving workload and performance was studied. The results of one-way ANOVA showed that there were significant differences in drivers' workload and performance under different test scenarios. In the $9^{\text {th }}$ test section, drivers not only showed a higher subjective workload score but also showed the characteristics of large speed fluctuation and unstable lane maintenance. This is a 
result of the $9^{\text {th }}$ test section being a sharp curve and steep slope section with a large traffic volume and too much road name information on signboards. Drivers not only have a high demand for information perception and processing but also require complex vehicle control on sharp curves and steep slope sections. Although the control strategy of reducing vehicle speed is adopted, some drivers still experience deteriorated driving performance. Multivariate analysis of variance of the orthogonal test indicated that all of the influencing factors have significant effects on the driver's workload and speed keeping ability, but driver's lane keeping ability was only significantly affected by the horizontal curve radius. The comprehensive evaluation of the correlation degree using the entropy weight method demonstrated that the amount of sign information has the greatest impact on the subjective workload, the traffic flow has the greatest influence on the mean and standard deviation of vehicle speed, and the radius of curve has the greatest effect on the mean and standard deviation of lane offset.

Note that since the research object is a freeway, it has the characteristics of full access control and a relatively simple traffic composition. Variable workloads caused by uncertain environments, such as traffic events, and their impact on driving behavior are not considered. Therefore, the established correlation degree model has a specific scope of application. For further research, we could introduce a comprehensive evaluation method such as the human factors analysis and classification system (HFACS) [48] to analyze the influencing factors from a systematic perspective, considering humans, vehicles, the environment, and management.

\section{Data Availability}

The data used to support the findings of this study are available from the corresponding author upon request.

\section{Conflicts of Interest}

The authors declare that there are no conflicts of interest regarding the publication of this paper.

\section{Acknowledgments}

The authors acknowledge the support from the National Nature Science Foundation of China (51775396), the National Nature Science Foundation of Guangxi Province (2019JJB160047), and Hubei Province Science Fund for Distinguished Young Scholars (2020CFA081).

\section{References}

[1] Ministry of Public Security Traffic Management Bureau, White Paper on Road Traffic Accidents in China 2019, Ministry of Public Security Traffic Management Bureau, Beijing, China, 2020.

[2] S. John, "Cognitive load during problem solving: effect on learning," Cognitive Science, vol. 12, no. 2, pp. 257-285, 1988.
[3] P. Julie, G. Edith, and B. Ca Therine, "Mental workload and driving," Frontiers in Psychology, vol. 5, 2014, http://www. ncbi.nlm.nih.gov/pmc/articles/PMC4251303/.

[4] H. Fuente, C. Berthelon, and A. Fort, "Electrophysiological and performance variations following driving events involving an increase in mental workload," European Transport Research Review, vol. 11, no. 1, 2019.

[5] J. Paxion, E. Galy, and C. Berthelon, "Mental workload and driving," Frontiers in Psychology, vol. 5, 2014 http://www.ncbi. nlm.nih.gov/pmc/articles/PMC4251303/.

[6] A. Dey and D. D. Mann, "Sensitivity and diagnosticity of NASA-TLX and simplified SWAT to assess the mental workload associated with operating an agricultural sprayer," Ergonomics, vol. 53, no. 7, pp. 848-857, 2010.

[7] N. Benguigui and F. Nicolas, "The effects of driving environment complexity and dual tasking on drivers' mental workload and eye blink behavior," Transportation Research Part F: Traffic Psychology and Behaviour, vol. 40, 2016.

[8] P. Choudhary, N. M. Pawar, N. R. Velaga, and D. S. Pawar, "Overall performance impairment and crash risk due to distracted driving: a comprehensive analysis using structural equation modelling," Transportation Research Part F: Traffic Psychology and Behaviour, vol. 74, pp. 120-138, 2020.

[9] T. Heine, G. Lenis, P. Reichensperger, T. Beran, O. Doessel, and B. Deml, "Electrocardiographic features for the measurement of drivers' mental workload," Applied Ergonomics, vol. 61, pp. 31-43, 2017.

[10] L. Neng-Chao, Y. Cao, L. Qin, and C.-Z. Wu, "Research on the effectiveness of driving workload based on traffic sign information volume," China Journal of Highway and Transport, vol. 31, no. 8, pp. 165-172, 2018.

[11] D. Baldauf, E. Burgard, and M. Wittmann, "Time perception as a workload measure in simulated car driving," Applied Ergonomics, vol. 40, no. 5, pp. 929-935, 2009.

[12] X. Chen, L. Qi, Y. Yang et al., "Video-based detection infrastructure enhancement for automated ship recognition and behavior analysis," Journal of Advanced Transportation, vol. 2020, Article ID 7194342, 12 pages, 2020.

[13] K. Ryu and R. Myung, "Evaluation of mental workload with a combined measure based on physiological indices during a dual task of tracking and mental arithmetic," International Journal of Industrial Ergonomics, vol. 35, no. 11, pp. 991-1009, 2005.

[14] H. Wiberg, E. Nilsson, P. Lindén, B. Svanberg, and L. Poom, "Physiological responses related to moderate mental load during car driving in field conditions," Biological Psychology, vol. 108, pp. 115-125, 2015.

[15] M. Zokaei, M. J. Jafari, R. Khosrowabadi, A. Nahvi, S. Khodakarim, and M. Pouyakian, "Tracing the physiological response and behavioral performance of drivers at different levels of mental workload using driving simulators," Journal of Safety Research, vol. 72, pp. 213-223, 2020.

[16] B. Wu, Y. H. Tang, Y. Tang, X. Yan, and C. Guedes Soares, "Bayesian Network modelling for safety management of electric vehicles transported in RoPax ships," Reliability Engineering \& System Safety, vol. 209, Article ID 107466, 2021.

[17] D. D. Heikoop, J. C. F. De Winter, B. Van Arem, and N. A. Stanton, "Effects of mental demands on situation awareness during platooning: a driving simulator study," Transportation Research Part F: Traffic Psychology and Behaviour, vol. 58, pp. 193-209, 2018.

[18] X. U. Jin and X. Wang, "Foot manoeuvres and workload of driver on mountainous roads with longitudinal slope," China 
Journal of Highway and Transport, vol. 31, no. 01, pp. 91-100, 2018.

[19] X. Li, X. Yan, and S. C. Wong, "Effects of fog, driver experience and gender on driving behavior on S-curved road segments," Accident Analysis \& Prevention, vol. 77, pp. 91-104, 2015.

[20] J. E. Hummer, W. Rasdorf, D. J. Findley, C. V. Zegeer, and C. A. Sundstrom, "Curve collisions: road and collision characteristics and countermeasures," Journal of Transportation Safety \& Security, vol. 2, no. 3, pp. 203-220, 2010.

[21] J. Hu, X. Li, and S. Luo, "The definition of long steep downgrade based on driver behavior," Transactions of Beijing Institute of Technology, vol. 37, no. 6, pp. 590-594, 2017.

[22] B. Farahmand and A. M. Boroujerdian, "Effect of road geometry on driver fatigue in monotonous environments: a simulator study," Transportation Research Part F: Traffic Psychology and Behaviour, vol. 58, pp. 640-651, 2018.

[23] B. Metz and H.-P. Krüger, "Do supplementary signs distract the driver?" Transportation Research Part F: Traffic Psychology and Behaviour, vol. 23, no. mar, pp. 1-14, 2014.

[24] J. Roca, C. Castro, M. Bueno, and S. Moreno-Ríos, “A drivingemulation task to study the integration of goals with obligatory and prohibitory traffic signs," Applied Ergonomics, vol. 43, no. 1, pp. 81-88, 2012.

[25] Y. Yang, Y. Chen, and C. Wu, "Effect of highway directional signs on driver mental workload and behavior using eye movement and brain wave," Accident Analysis \& Prevention, vol. 146, 2020.

[26] J. Edquist, C. M. Rudin-Brown, and M. G. Lenné, “The effects of on-street parking and road environment visual complexity on travel speed and reaction time," Accident Analysis \& Prevention, vol. 45, pp. 759-765, 2012.

[27] Y. Yan-Qun, T. Qiao, and Z. Xin-Yi, "Effectiveness analysis of freeway guide signs based on DEA model," China Journal of Highway and Transport, vol. 1-17, 2020, http://kns.cnki.net/ $\mathrm{kcms} /$ detail/61.1313.u.20200415.1231.004.html.

[28] X. Chen, Z. Li, Y. Yang, L. Qi, and R. Ke, "High-resolution vehicle trajectory extraction and denoising from aerial videos," IEEE Transactions on Intelligent Transportation Systems, vol. 22, no. 5, pp. 3190-3202, 2021.

[29] K. Fitzpatrick, S. T. Chrysler, E. S. Park, V. Iragavarapu, and A. A. Nelson, "Driver performance at high speeds using a simulator," Transportation Research Record: Journal of the Transportation Research Board, vol. 2321, no. 1, pp. 88-97, 2012.

[30] N. Lyu, Y. Cao, C. Wu, J. Xu, and L. Xie, "The effect of gender, occupation and experience on behavior while driving on a freeway deceleration lane based on field operational test data," Accident Analysis \& Prevention, vol. 121, no. 121, pp. 82-93, 2018.

[31] D. De Waard, A. Kruizinga, and K. A. Brookhuis, "The consequences of an increase in heavy goods vehicles for passenger car drivers' mental workload and behaviour: a simulator study," Accident Analysis \& Prevention, vol. 40, no. 2, pp. 818-828, 2008.

[32] S. Tement, N. Plohl, M. Horvat, B. Musil, G. Jakus, and J. Sodnik, "Driving demands, stress reactivity and driving behavior: an interactional approach," Transportation Research Part F: Traffic Psychology and Behaviour, vol. 69, pp. 80-90, 2020.

[33] R. Fuller, "The task-capability interface model of the driving process," Recherche Transports Sécurité, vol. 66, pp. 47-57, 2000.
[34] K. A. Brookhuis and D. De Waard, "Monitoring drivers' mental workload in driving simulators using physiological measures," Accident Analysis \& Prevention, vol. 42, no. 3, pp. 898-903, 2010.

[35] L. Xie, C. Wu, N. Lyu, and Z. Duan, "Studying the effects of freeway alignment, traffic flow, and sign information on subjective driving workload and performance," Advances in Mechanical Engineering, vol. 11, no. 5, Article ID $168781401985369,2019$.

[36] W. Gao and X. Gao, "An analysis of influencing factors of workload of air traffic controllers based on weighted gray correlation degree," Journal of Transport Information and Safety, vol. 34, no. 3, pp. 108-115, 2016.

[37] G. Borghini, L. Astolfi, G. Vecchiato, D. Mattia, and F. Babiloni, "Measuring neurophysiological signals in aircraft pilots and car drivers for the assessment of mental workload, fatigue and drowsiness," Neuroscience \& Biobehavioral Reviews, vol. 44, pp. 58-75, 2014.

[38] L. Y. U. Nengchao, L. Xie, and C. Wu, "Driver's cognitive workload and driving performance under traffic sign information exposure in complex environments: a case study of the highways in China," International Journal of Environmental Research and Public Health, vol. 14, p. 203, 2017.

[39] D. Xu, Y. Yin, X. Zhang, T. Sun, and Y. E. Jin, "Grey analysis and prediction of maritime traffic accidents on the trunk line of Yangtze River," Navigation of China, vol. 42, no. 002, pp. 59-65, 2019.

[40] W. Tao, Y. Chen, and X. Yan, “Assessment of drivers' comprehensive driving capability under man-computer cooperative driving conditions," IEEE Access, vol. 8, no. 99, p. 1, 2020.

[41] G. M. Fitch, K. Grove, R. J. Hanowski, and M. A. Perez, "Compensatory behavior of drivers when conversing on a cell phone investigation with naturalistic driving data," Transportation Research Record, vol. 2434, no. 1, pp. 1-8, 2014.

[42] T. Ben-Bassat and D. Shinar, "The effect of context and drivers' age on highway traffic signs comprehension," Transportation Research Part F: Traffic Psychology and Behaviour, vol. 33, pp. 117-127, 2015.

[43] N. Lyu, C. Deng, and L. Xie, "A field operational test in China: exploring the effect of an advanced driver assistance system on driving performance and braking behavior," Transportation Research Part F: Traffic Psychology and Behaviour, vol. 65, Article ID S1369847816306672, 2018.

[44] C. Dijksterhuis, K. A. Brookhuis, and D. De Waard, "Effects of steering demand on lane keeping behaviour, self-reports, and physiology. A simulator study," Accident Analysis \& Prevention, vol. 43, no. 3, pp. 1074-1081, 2011.

[45] K. L. Young, R. Osborne, and R. Grzebieta, "Using naturalistic driving data to examine how drivers share attention when engaging in secondary tasks," Safety Science, vol. 129, pp. 1-9, 2020.

[46] J. Engström, G. Markkula, T. Victor, and N. Merat, "Effects of cognitive load on driving performance: the cognitive control hypothesis," Human Factors: The Journal of the Human Factors and Ergonomics Society, vol. 59, no. 5, pp. 734-764, 2017.

[47] T. Chen, N. N. Sze, and S. Newnam, "Effectiveness of the compensatory strategy adopted by older drivers: difference between professional and non-professional drivers," Transportation Research Part F: Traffic Psychology and Behaviour, vol. 77, no. 2, 2021.

[48] D. Jiang, B. Wu, Z. Cheng, J. Xue, and P. H. A. J. M. Van Gelder, "Towards a probabilistic model for estimation of grounding accidents in fluctuating backwater zone of the Three Gorges Reservoir," Reliability Engineering \& System Safety, vol. 205, Article ID 107239, 2021. 